\title{
Dependence of De-molding Force on Process Conditions in UV Nanoimprint
}

\author{
Tomoki Nishino $^{1,2}$, Ryousuke Suzuki ${ }^{1,2}$, Hiroaki Kawata ${ }^{1,2}$ and Yoshihiko Hirai ${ }^{1,2}$ \\ ${ }^{\prime}$ Osaka Prefecture University \\ Department of Physics and Electronics Engineering, Graduate School of Engineering \\ 1-1 Gakuen-cho, Nakaku, Sakai, Osaka, 599-8531 Japan \\ ${ }^{2}$ Japan Science and Technology Agency \\ CREST (Core Research of Evolutional Science \& Technology) \\ 4-1-8, Honcho, Kawaguchi-shi, Saitama 332-0012 Japan
}

\begin{abstract}
The de-molding force between a UV resist and a Si dioxide surface is measured for various UV light intensities and exposure times. Typical UV radical polymerization resists are examined without any surface treatment of the $\mathrm{Si}$ dioxide surface for releasing. The result shows that the de-molding force increases in as increments of the UV light intensity, however it does not depend on the UV dosage. But the de-molding force depends on the product of the square root of the light intensity and exposure time, which is nearly consistent with the UV curing characteristics of the polymer.
\end{abstract}

Keywords: UV nanoimprint, UV curable resist, de-molding force, light intensity, dosage

\section{Introduction}

Nanoimprint lithography [1] is expected to produce fine patterns for advanced devices at efficient costs. UV nanoimprint is especially applied for such industrial applications as subwavelength optical elements or patterned media because the total turnaround time is shorter than for the thermal nanoimprint process.

In the UV nanoimprint process, one issue is the de-molding process that affects the defect yield. Even though the UV curing properties, including the polymer's adhesive characteristics, are essential for de-molding, there are few reports on de-molding characteristics related to VU curing characteristics, such as UV light intensity or exposure time.

Our previous report investigated the relations between the UV curing characteristics of radical UV resists and exposure light intensity [2]. Monomer consumption and modulus modification are fairly expressed by the product of the square root of the light intensity and the exposure time.

This paper investigates C-TGC-02 and
C-TGC-03 resists (Toyo Gosei), which are pilot UV radical resists. The de-molding force between the resist and the $\mathrm{SiO}_{2}$ surface is measured for various UV light intensities, and the results are compared to such UV curing characteristics as the monomer conversion ratio.

\section{Experiment}

There are two types of UV cable resists for nanoimprint lithography: a radical polymerization type and an ion polymerization type. In this study, we examined C-TGC-02 and C-TGC-03 resists, which are pilot UV radical resists that consist of crylic acid adduct of propyleneglycol diglycidyl ether/2 methyl-1(4-methylthiophenyl)-2-morpholino-propane-1-one produced by Toyo Gosei.

Figure 1 show the schematics of the sample. On a quartz plate, the UV resist is spin-coated using a primer to strongly ahead it to the plate. Then a $\mathrm{Si}$ tip with a $\mathrm{SiO}_{2}$ thin layer is put on the resist, where the $\mathrm{SiO}_{2}$ layer is grown by piranha boiling $\left(\mathrm{H}_{2} \mathrm{SO}_{4}\right.$ $+\mathrm{H}_{2} \mathrm{O}_{2}$ ) for 30 minutes. The $\mathrm{Si}$ tip is $10 \mathrm{~mm}$

Received April 11, 2011

Accepted May 13, 2011 
squared and gulled on an Al base.

Then UV light is irradiated to the resist through the quartz plate to cure it. To eliminate the effects of a release agent and the friction between a resist and a mold pattern, a flat $\mathrm{SiO}_{2}$ surface on a $\mathrm{Si}$ substrate is used. The arial distribution of the UV light intensity is measured, and the deviation is kept less than $10 \%$ of the $10 \mathrm{~mm}$ squared.

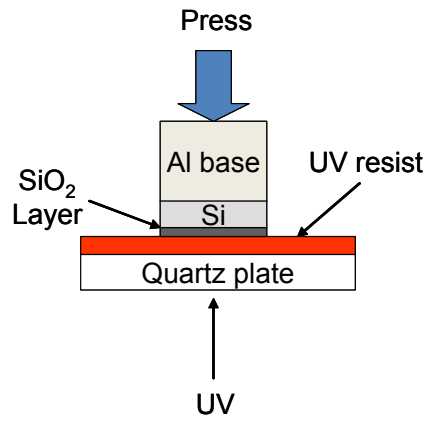

Figure 1 Schematic of sample preparation

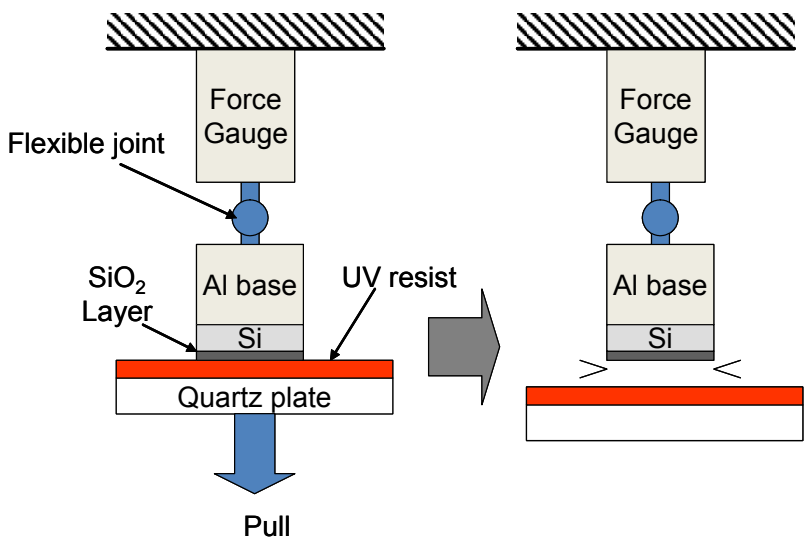

Figure. 2 Schematic of de-molding force measurement

Figure 2 show the schematic of the measurement of the de-molding force. The force gauge is connected to the Al base through a flexible joint and its position is fixed. The quartz plate is pulled down by a stepping motor at $0.1 \mathrm{~mm} / \mathrm{s}$. The de-molding force is measured by the force gauge when the resist is released from the $\mathrm{SiO}_{2}$ surface. The de-molding force per unit area of the Si tip is evaluated as the de-molding pressure.

\section{Results and discussions}

3.1 De-molding force measurement

Figures 3 and 4 show the measured de-molding force per unit area in variation with UV light intensity I for C-TGC-02 and C-TGC-03 resists. The intensities of the UV lights are 1, 3, and 30 $\mathrm{mW} / \mathrm{cm}^{2}$. As the UV irradiation time increases, the de-molding force also increases. As the increment of the UV intensity increases, the increment of the de-molding force is accelerated.

\subsection{Relation to polymerization of resists}

To investigate the relations between the de-molding force and the polymerization of resists by UV irradiation, we evaluated the dependence of the de-molding force on the exposure dosages. Figures. 5 and 6 show the relations between the de-molding force per unit area and the exposure dosages for various UV light intensities. The de-molding forces not only depend on the UV exposure dosages but also on the UV light intensity.

The chemical reaction speed of the UV radical polymerization resist depends on the products of the square root of UV intensity $I$ and exposure time $t$ [2]. Figures. 7 and 8 show the relations between the de-molding force per unit area and $\sqrt{I} t$ for various UV light intensities. The de-molding forces are fairly expressed by the value of $\sqrt{I} t$. This means that the de-molding force is nearly related to the polymer's UV curing characteristics.

To compare these phenomena with the chemical reactions of the resists, monomer conversion ratios were evaluated by FTIR (Figs. 9 and 10). The increment of the de-molding forces was delayed more than the monomer conversion by UV radical polymerization.

\section{Conclusion}

The de-molding force on various UV light intensities and exposure times was experimentally investigated using UV radical polymerization resists. The de-molding force is related to the product of the square root of the light intensity and the exposure time, which is related to the monomer conversion of the resists. 


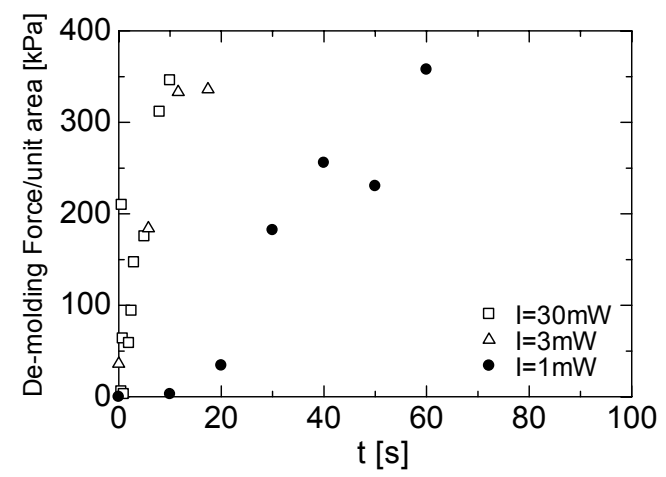

Figure 3 De-molding force for C-TGC-02

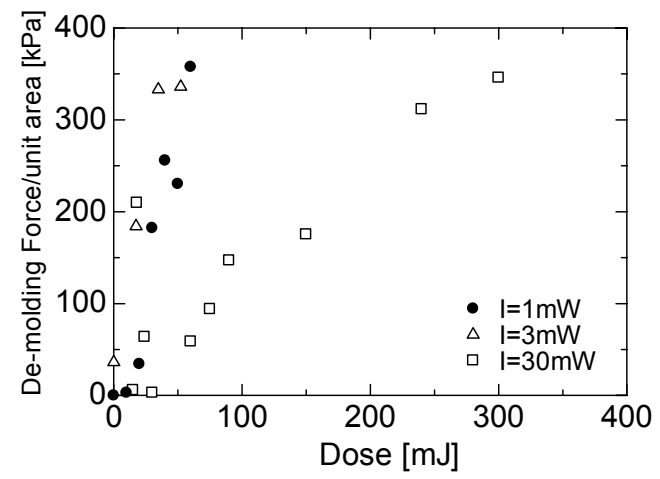

Figure 5 Dependence on dosage for C-TGC-02

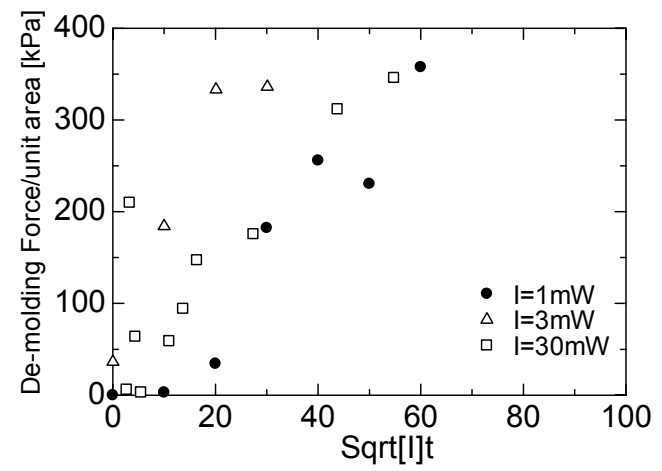

Figure 7 Dependence on $\sqrt{I} t$ for C-TGC-02

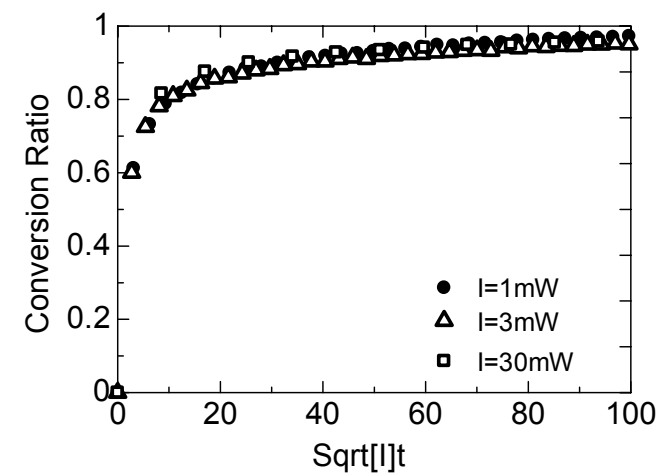

Figure 9 Monomer conversion for C-TGC-02

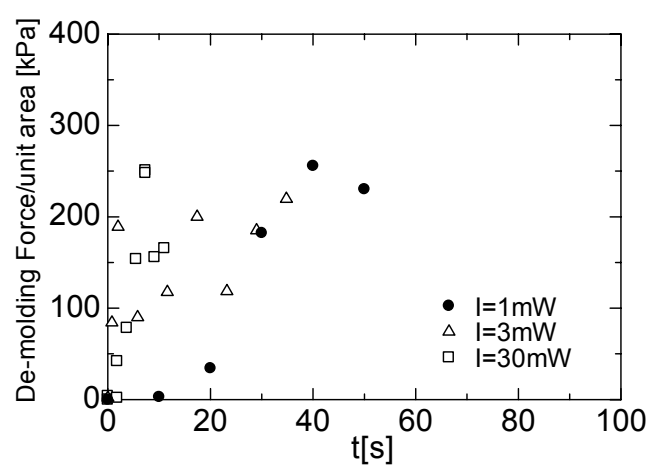

Figure 4 De-molding force for C-TGC-03

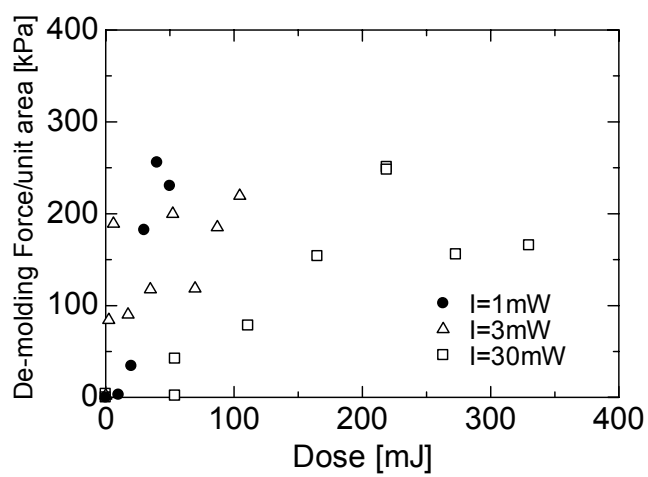

Figure 6 Dependence on dosage for C-TGC-03

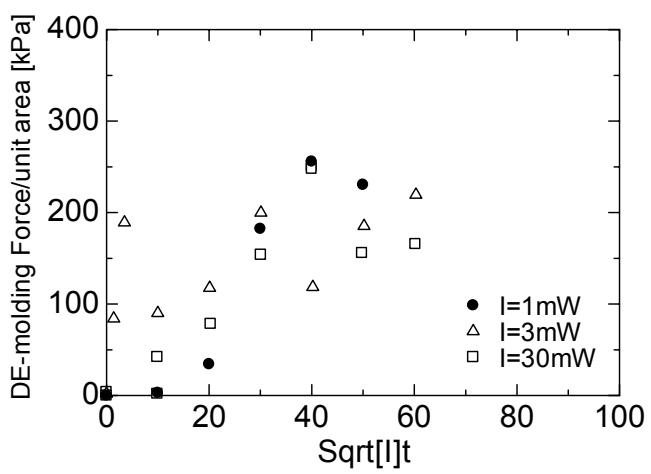

Figure 8 Dependence on $\sqrt{I} t$ for C-TGC-03

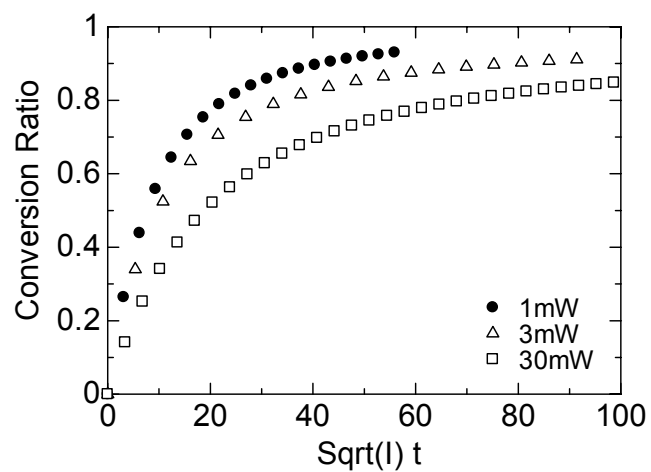

Figure 10 Monomer conversion for C-TGC-03 


\section{Acknowledgement}

The authors thank Mr. Osachi at Toyo Gosei Co. Ltd. for supplying the pilot UV radical polymerization resists.

\section{References}

1) M. Colburn, S. Johnson, M. Stewart, S. Damle, T. Bailey, B. Choi, M. Wedlake, T. Michaelson, S. V. Sreenivasan, J. Ekerdt, and C. G. Willson, Proc. SPIE 3676 (1999) 378.

2) R. Suzuki, N. Sakai, A. Sekiguchi, Y. Matsumoto, R. Tanaka and Y. Hirai, $J$. Photopolymer Sci. and Technol, 23 (2010)51.

3) F. A. Houle, E. Guyer, D. C. Miller, and R. Dauskardt, J. Vac. Sci. Technol. B $\mathbf{2 5}$ (2007)1179.
4) X. Ye, Y. Ding, Y. Duan, H. Liu, and B. Lu, $J$. Vac. Sci. Technol. B 27 (2009)2091.

5) J. Taniguchi, T. Kawasaki, Y. Tokano, Y. Kogo, I. Miyamoto, M. Komuro, H. Hiroshima, N. Sakai, and K. Tada, Jpn. J. Appl. Phys. 41 (2002) 4194.

6) V. Trabadeloa, H. Schifta, S. Merinob, S. Bellinic and J. Gobrechta, Microelectronic Engineering. 85 (2008) 907.

7) E. K. Kim, N. A. Stacey, B. J. Smith, M. D. Dickey, S. C. Johnson, B. C. Trinque, and C. G. Willson, J. Vac. Sci. Technol. B 22 (2004) 131. 